\title{
Öffentliche Mobilität finanzieren: Plädoyer für ein neues Verständnis der Mobilitätsfinanzierung im öffentlichen Verkehr
}

\section{Oliver Mietzsch}

\section{$1 \quad$ Einleitung}

Mit der Herausbildung integrativer Planungsansätze zur besseren Erfassung des Phänomens stellt sich nicht nur die Frage nach geeigneten Messinstrumenten zur Beschreibung der Komplexität der Bewegung von Menschen in Raum und Zeit. Vielmehr ergeben sich daraus auch gänzlich neue Anforderungen an den Bereich der Governance, d. h. die immateriellen und materiellen Rahmenbedingungen von Mobilität. $\mathrm{Zu}$ den immateriellen Rahmenbedingungen gehören die im historischen Kontext verorteten ethischen Begründungen für die Organisation von Mobilität ebenso wie die daraus entstandenen Handlungsmuster und Routinen, d. h. die Mobilitätskultur (siehe den Beitrag von Hoor in diesem Band). Davon nicht losgelöst sind die materiellen Rahmenbedingungen, d. h. die rechtliche Verfasstheit, organisatorische Zuständigkeitsverteilung und das daraus abgeleitete Finanzierungsinstrumentarium von Mobilität. Im Folgenden sollen diese Zusammenhänge am Beispiel der Öffentlichen Mobilität näher beleuchtet und daraus schlussfolgernd Vorschläge für eine andere Finanzierung von öffentlichem Verkehr unterbreitet werden.

\section{Mobilität und Verkehr}

Die öffentliche Hand ist der Hauptfinanzier der urbanen Mobilität. Dies gilt hinsichtlich des Betriebs (insbesondere beim öffentlichen Verkehr) und in noch weit größerem Umfang im Hinblick auf die Verkehrsinfrastruktur. Während die

O. Mietzsch $(\bowtie)$

Zweckverband für den Nahverkehrsraum Leipzig, Leipzig, Deutschland

E-Mail: mietzsch@zvnl.de 
Finanzierung der Betriebskosten von Straßenbahnen, U- und Stadtbahnen oder Bussen häufig im Rahmen des kommunalwirtschaftlichen Querverbunds (Verrechnung der Gewinne und Verluste der öffentlichen Versorgungsunternehmen) stattfindet, werden die Verkehrswege (Straßen, Gleis- und Betriebsanlagen des ÖPNV) direkt aus dem Steueraufkommen von Bund (Gemeindeverkehrsfinanzierungsgesetz), Ländern (Entflechtungsmittel) und Kommunen (z. B. im Rahmen des kommunalwirtschaftlichen Querverbunds) finanziert. Der Anteil der Finanzierung durch die Nutzer*innen der kommunalen Straßeninfrastruktur ist in Deutschland sehr übersichtlich (neben Einnahmen aus Anwohner*innenparkregelungen sind hier vor allem Sondernutzungsgebühren für die Nutzung von Gehwegen sowie Parkgebühren zu nennen. ${ }^{1}$ Demgegenüber liegt die Zahl der Fahrgeldeinnahmen der Fahrgäste des ÖPNV im Durchschnitt bei rund 75,6\% (VDV 2018). Dies führt jedoch nicht unbedingt zu einer geringeren finanziellen Beteiligung der zuständigen Gebietskörperschaften, da es mehrere Förderinstrumente gibt, die wie z. B. die Ausgleichsleistungen für den rabattierten Schüler- und Ausbildungsverkehr, die Schwerbehindertenfreifahrt, ermäßigte Tickets für sozial Benachteiligte oder zum Ausgleich von Einnahmeverlusten der Verkehrsunternehmen im Rahmen der Tarifintegration innerhalb von Verkehrsund Tarifverbünden, weitgehend aus öffentlichen Mitteln finanziert werden. Für die Betriebskosten der Regionalzüge zahlt der Bund jährlich einen Ausgleich von derzeit rd. 8,4 Mrd. € an die Länder, die selbst entweder direkt oder über lokale oder regionale Verkehrsorganisationen die Bahnbetreiber im Wege der öffentlichen Ausschreibung beauftragen. Infolgedessen ist das System der öffentlichen Finanzierung des ÖPNV hierzulande im internationalen Vergleich eher intransparent und komplex.

Allzu oft wird von Mobilität gesprochen, gemeint ist aber der Verkehr. Während der Mobilitätsbegriff die Möglichkeiten adressiert, die einem Individuum zur Überwindung von Raum und Zeit zur Verfügung stehen können, beschreibt der Begriff Verkehr die tatsächlich realisierten Ortsveränderungen. Damit stellt der Verkehr die abhängige Variable dar, d. h. ohne die konkrete Möglichkeit zur Überwindung von Raum und Zeit bleibt der Mobilitätsbegriff

\footnotetext{
${ }^{1} 30,70 €$ darf ein Parkausweis für ein Auto in Deutschland pro Jahr 1t. GebOSt höchstens kosten. Der Blick ins Ausland zeigt, dass die deutschen Parkgebühren sehr moderat sind. So bezahlen Anwohner*innen in Paris $45 €$ pro Jahr (und für drei Jahre $90 €$ ). Elektroautos oder gasbetriebene Fahrzeuge sind davon ausgenommen. In London beträgt diese Gebühr (165 €), in Amsterdam (535€) und in Stockholm (827 €). Vgl. https://www.agora-verkehrswende.de/fileadmin/Projekte/2017/Parkraummanagement/Parkraummanagemet-lohnt-sich_ Agora-Verkehrswende_web.pdf. Zugegriffen: 11.08.2020.
} 
eine hohle Floskel. Umgekehrt gilt jedoch, dass eine Fixierung auf die realisierten Ortsveränderungen ohne eine Betrachtung des Kontextes, indem diese stattfinden, nur eine weitere Facette des Positivismus darstellt. ${ }^{2}$ Sinnfällig wird dieser Widerspruch in der tagespolitischen Debatte um die Verteilung der begrenzten finanziellen Ressourcen. Die Vertreter*innen eines umfassenden Mobilitätsbegriffs verweisen unter Bezugnahme auf die Herausforderungen, die der Klimawandel sowie die demographische Entwicklung an die Organisation und Finanzierung von Verkehr stellt, darauf, dass die Verkehrsträger des öffentlichen Verkehrs, d. h. vor allem Bus und Bahn, gestärkt werden müssen zu Lasten des motorisierten Individualverkehrs (MIV). Demgegenüber beharren die Apologeten des „Juste Millieus“3 auf den derzeit vorherrschenden Mobilitätsmustern mit der Dominanz des Kfz als allein entscheidender Allokationsregel. Unbeachtet bleibt dabei aber allzu oft die Frage, woher überhaupt die Mittel kommen, die zur Sicherung der Mobilität, ob nun individuell oder kollektiv, benötigt werden. So wie das geflügelte Zitat vom Strom, der aus der Steckdose kommt, nur die halbe Wahrheit widerspiegelt, so wenig stammt die gesamte Finanzierung des Verkehrs nur aus Steuermitteln. Und selbst wenn das gesamte Steueraufkommen von Bund, Ländern und Kommunen ausreichen würde, um alle Mobilitätsbedarfe zu befriedigen, so stellt sich immer noch die Frage, ob dies auch so sein sollte. Wer Mobilität und Verkehr kategorial trennt, erkennt den Unterschied: Bei der Mobilitätsfinanzierung handelt es sich um die materielle Absicherung von Handlungsmöglichkeiten, d. h. verkehrlichen Optionen, während die Finanzierung des Verkehrs, d. h. die zu seiner Abwicklung erforderliche Infrastruktur und Betriebsmittel, bereits eine Wahlentscheidung für einen bestimmten Verkehrsträger

\footnotetext{
${ }^{2}$ Bezeichnung für eine Position, der zufolge sich die Realwissenschaften auf die Erforschung der beobachtbaren Sachverhalte („Positiva“) beschränken sollen. Begründer ist Hume; der Name selbst geht auf Comte zurück, der das Drei-Stadien-Gesetz der wissenschaftlichen Entwicklung formulierte: 1) Theologische Stufe: Reale Erscheinungen werden auf das Wirken eines Gottes oder mehrerer Götter zurückgeführt. 2) Metaphysische Stufe: Erfassung des allg. Wesens der Dinge (Essentialismus). 3) Positive Stufe (höchste): Beschreibung von Tatsachen. Als naiv-positivistische Position kann ein radikaler Empirismus interpretiert werden; seine Merkmale bestehen in der bloßen Akkumulation von Fakten und der Formulierung erfahrungsnaher Hypothesen, während die Suche nach allg. Theorien vernachlässigt wird. Vgl. https://wirtschaftslexikon.gabler.de/definition/positivismus-46391. Zugegriffen: 03.08.2020.

${ }^{3}$ Zum Bedeutungswandel des Begriffs aus aktuellem Anlass vergleiche Bednarz, L. ,Wie die Moralkritik zur Pose wird“ Der Tagesspiegel, https://causa.tagesspiegel.de/kolumnen/ liane-bednarz/wie-die-moralkritik-zur-pose-wird.html. Zugegriffen: 01.08.2020.
} 
voraussetzt. Letzteres nur aus Steuern zu finanzieren, bedeutet einer ökonomisch und ökologisch ineffizienten Angebotsplanung das Wort zu reden, ohne Rücksicht darauf, ob die von allen finanzierten Verkehrswege auch wirklich allen nützen.

\section{$3 \quad$ Nutznießerfinanzierung als neuer Ansatz - politische und theoretische Begründung}

Im MIV ist das Instrument der Nutznießerfinanzierung bereits seit langem bekannt: Bei Gemeinde- und Stadtstraßen können die Kommunen zur erstmaligen Herstellung (Erschließung) sowie zum Ausbau bzw. der Verbesserung oder grundhaften Erneuerung bzw. nachmaliger Herstellung Beiträge nach den Bestimmungen des § 127 BauGB bzw. den Kommunalabgabengesetze der Länder (KAG) erheben. Anknüpfungspunkt ist in beiden Fällen das Grundstück, d. h. in dem Umfang, in dem das Grundstück eines Anliegers einer solchen Straße an den Erschließungs- bzw. Ausbaumaßnahmen partizipiert, erfolgt auch die finanzielle Beteiligung. Die rechtliche Begründung dafür findet sich im sog. Gemeingebrauch $^{4}$, d. h. in dem Recht von jedermann zur Nutzung der Straße: „Der Inhalt des Gemeingebrauchs im Einzelnen unterliegt im Laufe der Zeit einem fortwährenden Wandel. Außer auf die Widmung kommt es auf die Gemeinüblichkeit an; die Gemeinverträglich dagegen, d. h. die Gewährleistung des störungsfreien Mitgebrauchs anderer, wird nicht als Element des Gemeingebrauchs gesehen, sondern als Begriff des Straßenverkehrsrechts" (Hegelau 1994, S. 16). M. a. W., zur Finanzierung eines Straßenneu- oder -ausbaus in einer Gemeinde können auch diejenigen herangezogen werden, die überhaupt kein eigenes $\mathrm{Kfz}$ besitzen oder dieses führen, da allein das Vorhandensein der Infrastruktur zu deren Nutzung befähigt.

Wenn das für die Nutzung der Straße gilt, warum dann nicht auch für die Nutzung von Bussen und Bahnen? Die Antwort ist simpel: Der kommunale

\footnotetext{
${ }^{4}$ Der Gemeingebrauch ist ein auf römisches Recht zurückgehender Rechtsbegriff (usus publicus), der heute noch vor allem im Straßenrecht Anwendung findet. Unter Gemeingebrauch ist das Recht einer Vielzahl von Menschen zur Benutzung solcher Sachen zu verstehen, die der Nutzung durch die Öffentlichkeit dienen - im Gegensatz zum Eigentums- und Besitzrecht, das die Nutzung von Sachen nur durch bestimmte einzelne Personen regelt. Im Gegensatz zum Eigentum ist er also kein Individualrecht, sondern ein Kollektivrecht. Das hat vor allem zur Folge, dass der zum Gemeingebrauch Berechtigte keinen anderen von der Nutzung ausschließen kann, der ebenfalls zum Gemeingebrauch berechtigt ist. Im Straßenrecht legt die Widmung den Rahmen der Benutzung fest.
} 
ÖPNV ${ }^{5}$ fehlt schlicht in der Definition von Erschließungsanlagen. Warum das so ist, darüber kann nur spekuliert werden. Wahrscheinlich fehlte Anfang der 70er Jahre des vorigen Jahrhunderts, als der Gesetzgeber erstmals auf die Automobilisierung und den damit einhergehenden sinkenden Fahrgastzahlen im öffentlichen Verkehr mit dem Gemeindeverkehrsfinanzierungsgesetz - GVFG als einem mineralölsteuerbasierenden Finanzierungsinstrument zum Ausbau der kommunalen Verkehrsinfrastruktur auch für den ÖV reagierte (Deutsch et al. 2016, S. 3), schlicht das Problembewusstsein bezüglich der Notwendigkeit einer dauerhaften Finanzierungsgrundlage für den kommunalen Verkehr. Diese fehlende Weitsicht, die sich übrigens auch in einem Ausblenden der mit der steuerfinanzierten Infrastrukturfinanzierung verbundenen Senkung des Raumwiderstands und als Reaktion hierauf dem Verlust von wohnungsnahen Zielen zeigt, ,was sich beispielsweise im Rückgang der Nahversorgungsmöglichkeiten besonders außerhalb der hochverdichteten Bereiche großer Städte widerspiegelt" (ebd.), veranlassten bereits Apel et al. (1995, S. 13) zu der Forderung, u. a. durch eine Erweiterung der Erschließungspflicht für den ÖPNV, die Abschaffung der Stellplatznachweispflicht für Kfz sowie weiteren wohnungspolitischen Maßnahmen und nicht zuletzt die Einführung einer Schwerverkehrsabgabe und die Änderung des Bundesverkehrswegeplans zu einer Flächensparenden und Verkehrsreduzierenden Siedlungs- und Verkehrsentwicklung beizutragen.

Während bis zur Mitte des 20. Jh. eher der Staat als ,natürlicher' Infrastrukturbereitsteller angesehen wurde, ist mit dem Aufkommen des sog. New Public Managements die staatliche Bereitstellung von Infrastruktur zunehmend unter Rechtfertigungsdruck geraten. Verantwortlich hierfür sind auch in Europa die sinkenden fiskalischen Handlungsmöglichkeiten der Staaten im Rahmen der Einhaltung der sog. Maastricht-Kriterien sowie generell die zunehmende Wettbewerbsorientierung im Europäischen Binnenmarkt (vgl. die Einleitung von Schwedes in diesem Band). Es ist allerdings kritisch zu hinterfragen, ob sich Infrastruktursysteme überhaupt eindeutig bestimmten Güterkategorien wie öffentlichen, d. h. von jedermann nutzbaren, oder privaten Gütern und somit proprietärem Gebrauch zuordnen lassen. Das Konzept des ,Klubguts‘ als

\footnotetext{
${ }^{5}$ In Abgrenzung zum Schienenpersonennahverkehr (SPNV), der die Verkehrsträger der schweren Schiene (heavy rail) umfasst und einem völlig anderen Rechts- und Finanzregime unterliegt, finanziert sich der kommunale straßengebundene ÖPNV (ÖSPV), hierzu gehören auch die spurgebundenen Systeme Straßen-, Stadt- und U-Bahnen sowie O-Busse, neben den Nutzerbeiträgen der Fahrgäste (Fahrgeldeinnahmen) primär aus dem allgemeinen Steueraufkommen.
} 
Mischform mit Eigenschaften aus privaten und öffentlichen Gütern beschreibt daher zutreffender leitungsgebundene (Verkehrs-)Infrastruktursysteme, die sich in sog. Netzeffekten wie der Erreichbarkeit der Haltepunkte, Wartezeiten sowie der Transportdauer manifestieren und denen hinsichtlich der Zahlungsbereitschaft sowie Konkurrenzfähigkeit im intermodalen Wettbewerb eine große Bedeutung zukommt. Vor diesem Hintergrund zeigt sich die Relevanz der Klubgütertheorie insbesondere bei der Finanzierung des öffentlichen Verkehrs (Lehmann und Rodi 1997, S. 11 ff.).

Der von der Nutzungselastizität abhängige Rivalitätsgrad bei Klubgütern lenkt den Blick auf die Bereitstellungskosten der Verkehrsinfrastruktur, welche wiederum die im ÖPNV-Markt vorhandenen positiven und negativen externen Effekte widerspiegeln. In der Finanzwissenschaft haben sich hierfür ,normative Grundsätze' herausgebildet. Als Stellschrauben zur Ausrichtung des Angebotes an ÖPNV-Verkehrsinfrastruktur an die Nutzungsintensität dienen vor diesem Hintergrund Preis (Nutzer-/Nutznießerentgelt), Klubgröße und/oder Bereitstellungsmenge (Kapazität) bzw. -güte (Qualität). Aufgrund des Umstandes, dass bei der schienengebundenen ÖPNV-Infrastruktur in Städten bis zur Erreichung einer bestimmten Kapazitätsgrenze keine Rivalität bei der Nutzung gegeben ist ${ }^{6}$, ist eine Kombination verschiedener Abgaben aus pauschalisierten Fixkostenbeiträgen und grenzkostenbasierten, von der individuellen Nutzung abhängigen Kostendeckungsbeiträgen sinnvoll. Diesem Mix aus fixen und flexiblen ÖPNVInfrastrukturbeiträgen entspricht im Bereich des (Straßen)beitragsrecht der Gemeindeanteil, d. h. der Anteil, der dem Durchgangsverkehr zugeschrieben wird und der daher nicht in die Beitragsfestsetzung einfließt. Hinsichtlich der ökonomischen Bedingungen führt die Ineffizienz eines Ausschlusses vom Gebrauch der ÖPNV-Infrastruktur automatisch zu der Frage der Erhebung von Nutzungsentgelten, d. h. deren Finanzierung über Zwangsabgaben. Hierfür bedarf es schon aus demokratietheoretischen Gründen einer staatlichen Regulierung (vgl. den Beitrag von Schwedes und Ringwald in diesem Band). Hierfür eignen sich in besonderer Weise die kommunalen Gebietskörperschaften.

\footnotetext{
${ }^{6}$ Sprungfixe Kosten sind Kosten, die innerhalb eines bestimmten Wertebereichs einer Kosteneinflussgröße konstant bleiben und sich diesseits und jenseits des Intervalls in ihrer Höhe sprunghaft ändern. Sie werden auf Änderungen der betrieblichen Kapazitäten zurückgeführt. So erhöhen sich z. B. durch die Erweiterung des Maschinenparks die Abschreibungen sprunghaft. https://www.wirtschaftslexikon24.com/d/sprungfixe-kosten/ sprungfixe-kosten.htm. Zugegriffen 07.08.2020.
} 
„Betrachtet man die Allokationsaufgaben der öffentlichen Hand und überlegt man, welche Finanzierungsmittel jeweils eingesetzt werden, kommt man zu einem erstaunlichen Ergebnis: Der Staat benötigt für diese Aufgaben - also vor allem die Bereitstellung der Infrastruktur - keine Steuern ..., sondern lediglich zweckgebundene Zwangsabgaben mit unterschiedlichen Adressatenkreisen“ (Grossekettler 1991, S. 18). Für Sander (1987, S. 151) liegt der Kernbereich der kommunalen Betätigung heutzutage in der Bereitstellung von Klubkollektivgütern und Gütern natürlicher Monopole. Dem entspricht der finanzverfassungsrechtliche Vorrang der Äquivalenzfinanzierung (Gebühren und Beiträge) vor einer Finanzierung der Kommunalaufgaben aus Steuern. In diesem Sinne dienen Gebühren bzw. im Falle des ÖPNV die Fahrgeldeinnahmen der Abgeltung der Zusatzkosten einer Nutzung, und decken daher in erster Linie die (betriebswirtschaftlichen) Grenzkosten der Bereitstellung ab. Gebühren, die über diese Grenzkosten hinausgehen, sind wohlfahrtsökonomisch Unsinn, weil dann Nutzer vom Gebrauch des Klub(kollektiv)gutes abgeschreckt würden, obwohl dieser grenzkostenfrei erzielbare Vorteile mit sich brächte. Allerdings ist der volkswirtschaftliche Kostenbegriff breiter gefasst als der betriebswirtschaftliche, sodass bei der Bepreisung des Klub(kollektiv)gutes schienengebundene ÖPNV-Infrastruktur auch der Nutzenverzicht in Form von Überfüllung, Überlastung bzw. Verdrängung berücksichtigt werden muss. Daher sind bei der Bepreisung der Infrastruktur sowohl die Nutzungselastizität, d. h. die freien Bedienungskapazitäten, als auch die Mengenelastizität, d. h. die sich aus der Unteilbarkeit der Infrastrukturanlagen ergebenden Kostendegressionen, zu berücksichtigen. Daraus kann in Anlehnung an Grossekettler (1991, S. 9) die Schlussfolgerung gezogen werden, dass von den Klubmitgliedern immer dann ein ergänzender (nutzungsabhängiger) Kostenbeitrag erhoben werden sollte, wenn der (betriebswirtschaftliche) Grenzkostenpreis nicht die vollen Kosten pro Mitglied deckt: „Dieser Beitrag kann als ein Optionspreis dafür angesehen werden, das jeweilige Gut zum Grenzkostenpreis nutzen zu dürfen (ebd.).“ Diesem Grundgedanken entspricht finanzverfassungsrechtlich der Beitrag, der der Finanzierung der übrigen, d. h. der Fixkosten dient, da er auf den Optionsnutzen abstellt. Auf den ÖPNV bezogen bedeutet dies, dass die Fahrgeldeinnahmen in erster Linie die Kosten der Infrastrukturbewirtschaftung abdecken, während die Beiträge der Nutznießer*innen von schienengebundenen ÖPNV-Infrastrukturanlagen vor allem der Deckung der Infrastrukturvorhaltekosten dienen. Knieps (2002, S. 11) spricht sich dafür aus, die Erträge aus der Nutzung der Netzkapazitäten zur Finanzierung der Netzinfrastruktur zu verwenden: „Es ist daher durchaus ökonomisch sinnvoll, das Allokationsproblem knapper Kapazitäten und das Problem der Finanzierung der Netzinfrastruktur in einem Zusammenhang zu stellen. Dies darf allerdings nicht 
zu dem Umkehrschluss verleiten, auf Infrastrukturen mit geringer Nachfrage die Kosten der Infrastruktur nutzungsabhängig zu allozieren, weil hierdurch die verbleibende Nachfrage völlig abgeschreckt würde.“

\section{$4 \quad$ Beiträge als Finanzierungsinstrument}

Im Unterschied zu Steuern - deren finanzverfassungsrechtlichen Kompetenzgrundlagen sich in Art. 105 ff. GG finden mit der überwiegenden Zuständigkeit beim Bund; die Länder haben eine Gesetzgebungskompetenz nur im Hinblick auf die örtlichen Aufwands- und Verbrauchssteuern - enthält das Grundgesetz keine ausdrückliche Regelung für Gebühren und Beiträge, die auch als Vorzugslasten bezeichnet werden. „Aufgrund des Entgeltcharakters der Gebühren und Beiträge wird das Gebühren- und Beitragsrecht seither von Rechtsprechung und Literatur als ,Annexrecht“ angesehen“ (Klusemann 1998, S. 29). Kompetenzgrundlage für die Vorzugslasten ist die jeweilige Sachmaterie. Die Kommunen sind somit auf die gesetzlich vorgeschriebenen Kommunalabgabenarten beschränkt, mithin dürfen nur Abgaben erhoben werden, die gesetzlich bestimmt oder bestimmbar sind. Ein eigenständiges kommunales Steuerfindungsrecht findet sich gemäß Art. 105 Abs. 2 A GG sowie dem darauf basierenden § 3 AO lediglich im Hinblick auf die sog. kleinen Gemeindesteuern bzw. Bagatellsteuern, die sich auf den örtlichen Aufwand bzw. Verbrauch beziehen.

Der Gesetzesvorbehalt für Kommunalabgaben konkretisiert sich z. B. bei Erschließungs- und Straßenbaubeiträgen dahin gehend, dass die Gemeinden eine Erhebungspflicht haben,. m. a. W.: eine beitragsfähige Straßenbaumaßnahme darf nicht beschlossen werden, ohne dass zuvor die für die Beitragserhebung erforderliche Beitragssatzung erlassen wurde. Nach der am 3. Oktober 1990 vollzogenen staatlichen Wiedervereinigung Deutschlands setzten Bundestag und Bundesrat Ende 1991 eine Gemeinsame Verfassungskommission ein, die gemäß Art. 5 des Einigungsvertrages vom 31. August 1990 über notwendige bzw. wünschenswerte Änderungen und Ergänzungen des Grundgesetzes beraten und Vorschläge unterbreiten sollte. Im Zusammenhang mit der schon damals geforderten, aber nur ansatzweise umgesetzten Föderalismusreform wurde das Erschließungsbeitragsrecht explizit aus dem Kanon der konkurrierenden Gesetzgebung des Art. 74 GG ausgenommen und somit der Gesetzgebungskompetenz der Länder zugeordnet, gleichzeitig wurde aber mit Art. 125a GG statuiert, dass Recht, welches als Bundesrecht erlassen worden ist, jedoch wegen nachträglicher Änderung des Grundgesetzes nicht mehr als Bundesrecht erlassen werden kann, 
weiterhin als Bundesrecht gilt. Es kann aber durch Landesrecht aufgehoben und ergänzt werden.

Strittig ist in diesem Zusammenhang, ob der Bund mit Wirkung für die Länder, die noch keine eigenen landesgesetzlichen Regelungen zur Erhebung von Erschließungsbeiträgen durch die Kommunen erlassen haben, das Erschließungsbeitragsrecht weiterhin verändern kann, z. B. im Hinblick auf eine Erweiterung des Anlagenbegriffs um den öffentlichen Personenverkehr. Einige Autoren vertreten die Auffassung, dass eine nachträgliche Änderung der diesbezüglichen bundesrechtlichen Vorschriften wegen einer faktischen Versteinerungswirkung, die durch die Überführung der §§ 127-135 BauGB, die bislang der konkurrierenden Gesetzgebung unterlagen, in den Bereich der ausschließlichen Gesetzgebungskompetenz der Länder einträte, nicht mehr möglich sei (Hilgers 2012, S. 5.) In der Konsequenz könne der Bundesgesetzgeber selbst eine diesbezügliche Erweiterung des Anlagenbegriffs nicht mehr vornehmen. Und die in $\S 127$ Abs. 4 BauGB als Option ausgestaltete Möglichkeit der Kommunen, Abgaben für Anlagen $\mathrm{zu}$ erheben, die nicht Erschließungsanlagen im Sinne dieses Abschnittes des BauGB seien, stelle keine eigenständige Rechtsgrundlage für eine Abgabenerhebung dar. Diese könne sich nur aus einem anderen Gesetz, insbesondere den allgemeinen Kommunalabgabengesetzen ergeben (Grziwotz 2012, § 127 Rdnr. 25).

Diese Sichtweise konfligiert jedoch mit der Bestimmung des Art. 125a Abs. 1 GG, die dem Bund über den Wortlaut hinaus die Möglichkeit der Fortschreibung der als Bundesrecht fortgeltenden Vorschriften in den Fällen eröffnet, in denen die Gesetzgebungskompetenz des Bundes u. a. wegen der Änderung des Art. 74 Abs. 1 GG nicht mehr besteht. Die Kompetenz des Bundes endet demnach erst, wenn ein Land von seiner neuen Gesetzgebungskompetenz Gebrauch gemacht hat - und zwar nur in Bezug auf dieses Land (Jarass und Pieroth 2007, Rdn. 7 zu Art. 125a GG). Diese Sichtweise entbehrt nicht einer gewissen Logik, da in den Fällen, in denen ein Land aus welchen Gründen auch immer von seinen neuen Kompetenzen (noch) keinen Gebrauch gemacht hat, der Bund ansonsten nicht in der Lage wäre, z. B. bei einer Änderung der Verhältnisse eine Anpassung der Gesetzeslage vorzunehmen. Insofern wird die Auffassung vertreten, dass - solange keine abweichenden landesgesetzlichen Regelungen getroffen worden sind - auch der Bundesgesetzgeber die Möglichkeit besitzt, den Anlagenbegriff des $\S 127$ BauGB zu verändern. Den Ländern bliebe es allerdings unbenommen, davon nach Erlass einer eigenen gesetzlichen Regelung des Erschließungsbeitragsrechts wieder Abstand zu nehmen (Heckmann 1997, S. 284 f.). 


\section{$5 \quad$ Verkehrliche Erschließungs- und Erreichbarkeitsparameter als Äquivalent zum straßenrechtlichen Gemeingebrauch}

Wenn der Gemeingebrauch als rechtliche Voraussetzung für die Erhebung von Straßenbeiträgen losgelöst von der konkreten Nutzung anerkannt ist, stellt sich die Frage, warum das im schienengebundenen ÖPNV nicht auch so sein kann. Der wesentliche Unterschied liegt in der Zugangsmöglichkeit. Eine Straße ist grundsätzlich von jedermann nutzbar, neben der Bedeutung als Verkehrsweg für den Individualverkehr dient sie u. a. auch dem Gütertransport und kann je nach Widmung auch eine Aufenthaltsfunktion innehaben. Demgegenüber nutzt eine Gleisanlage bzw. Trasse ohne einen Betrieb auf derselben niemandem, sodass schon aus sicherheitstechnischen Gründen ein Zugang nur an hierzu bestimmten baulichen Anlagen (Haltepunkte, Verkehrsstationen) möglich ist. Weiterhin ist zu beachten, dass im Vergleich zum Straßenbau bei der Erschließung mit ÖPNV mit einem weitaus höheren Anteil laufender Kosten und damit höheren Folgekosten für die Gemeinde und - im Gegensatz zu Erschließungsanlagen der Ver- und Entsorgung - bei einer Ausweiterung der Erschließungspflicht auf den ÖPNV nicht mit einer gesicherten Nutzung der zusätzlich geschaffenen Angeboten gerechnet werden kann (Apel et al. 1995, S. 93). Apel et al. (1995, S. 94) plädieren daher dafür, dass Erschließungsanlagen für den ÖPNV und den MIV nicht getrennt voneinander betrachtet werden und dabei auch berücksichtigt wird, dass Mehraufwendungen für den ÖPNV a priori zu Lasten der Gemeinde gehen, „,während damit gleichzeitig verbundene Einsparungen beim motorisierten Individualverkehr zumindest in Bezug auf verminderten Stellplatzbedarf und geringere KfzBetriebskosten die Privaten begünstigen“.

Aber auch dann ist die Nutzbarkeit der ÖV-Anlage ohne ein konkretes Verkehrsangebot noch nicht automatisch gegeben.

„Der Anschluss an das öffentliche Nahverkehrsnetz ist für denjenigen nutzbringend, dem dadurch eine adäquate verkehrliche Alternative bei der Bewältigung seiner regelmäßig zurückzulegenden Wegstrecken geboten wird. Dieser Fall tritt jedoch nicht automatisch mit der Errichtung eines Haltepunktes (o. ä.) in Wohnortnähe ein. Ein solcher ,Anschluss` an den öffentlichen Verkehr reicht für sich genommen als Beförderungsoption nicht aus, da es sich beim Verkehr nicht um eine standortbezogene, sondern eine vektorielle (gerichtete) Größe handelt“ (Maak 1998, S. 147). ${ }^{7}$

\footnotetext{
${ }^{7}$ Der Autor bezieht sich dabei auf den Abdruck des Vortrags von Gerd Steierwald; Aspekte der regionalen Verkehrsentwicklung, bei der Verbandsversammlung des Regionalverbandes Stuttgart am 17.04.1991, S. 16.
} 
Demnach zeichnen sich alle regelmäßig zu bewältigenden Wegstrecken sowohl durch einen Ausgangs- (Quelle) als auch einen Endpunkt (Ziel) aus. Nur wenn beide Punkte über eine adäquate direkte Verbindung vom öffentlichen Verkehr angefahren werden, kann von einer zusätzlichen, nutzbringenden Verkehrsalternative gesprochen werden. „Der Anschluss des Wohnortes an das öffentliche Verkehrsnetz deckt zunächst nur die Verkehrsquelle ab. Liegt jedoch der regelmäßige Zielort außerhalb des bedienten Bereichs, tendiert der Wert der Verkehrseinrichtung für den konkreten Fall gegen Null. Die potentielle Nutzung des öffentlichen Verkehrs hängt also nicht nur vom Wohnstandort, sondern auch im selben Maße vom Fahrziel ab“ (ebd). Darin unterscheidet sich der öffentliche Verkehr und allzumal der schienen- bzw. spurgebundene ÖPNV wesentlich vom Individualverkehr. „Wesentliches Merkmal der Eisenbahn (diese gilt hier als pars pro toto für alle spurgeführten Verkehrssysteme, O.M.) ist [...] die technische Einheit von Fahrweg und Verkehrsmittel. Dieses ,maschinelle Ensemble', das keine spontanen Ausweichmöglichkeiten bietet, ist nur sehr eingeschränkt für den Individualverkehr geeignet" (Schmitz 2001, S. 35).

Es bedarf somit sowohl quantitativ zu messender Parameter der Erreichbarkeit im Sinne der Zugänglichkeit zum ÖPNV-System z. B. in Form der fußläufigen Entfernung zum nächsten ÖPNV-Haltepunkt als auch qualitativ zu beschreibender Parameter im Hinblick auf die Güte einer bestimmten Erschließung, die sich von diesem Haltepunkt im öffentlichen Nahverkehrsnetz bietet (Köhler 2011, S. 666). ${ }^{8}$ Beide Parameter beziehen sich auf die Überwindung von Raum und Zeit und sind daher im Kontext der gesellschaftlichen und Siedlungsentwicklung zu beleuchten. Interessanterweise haben sich dabei das Reisezeitbudget, also die zur Raumüberwindung erforderliche Menge an Zeit, nicht wesentlich verändert, sondern insbesondere die Entfernungen. In der Verkehrswissenschaft wird dies als „Konstanz des Reisezeitbudgets“ bezeichnet (Knoflacher 2016, S. 630).

Vor diesem Hintergrund kommt Schmitz (2001, S. 189) zur Definition von verkehrlich wirksamen Handlungsmustern, die sich aus der Erweiterung der

\footnotetext{
${ }^{8}$ Der Autor beschreibt die Erreichbarkeit eines Zielortes von einem Quellort aus sowohl über die Reisezeit mit einem bestimmten Verkehrsmittel als auch über die Zahl der Aktivitätengelegenheiten. Zur Messung der Qualität der Erreichbarkeit werden mindestens zwei Verkehrsmittel (i. d. R. ein individuelles und ein öffentliches Verkehrsmittel) hinsichtlich der Reisezeit miteinander verglichen. Die Bewertung der Erreichbarkeitsqualität erfolgt dann über den Vergleich der absoluten Reisezeiten beider Verkehrsmittel zwischen Quellund Zielort, dem Reisezeitverhältnis der beiden Verkehrsmittel zueinander sowie der relativen Ausstattung des Zielortes mit Gelegenheiten.
} 
Aktionsreichweiten infolge höherer Geschwindigkeiten bei gleichbleibendem Reisezeitbudget ergeben. Die erste Kategorie besteht demzufolge in der Wahl der Aktionsräume (Haushalte) und Marktgebiete (Unternehmen) sowie der damit zusammenhängenden räumlichen Interaktionen, wodurch sich die Wahlfreiheit sowohl im Hinblick auf längerfristig angelegte Aktivitätsorte wie z. B. Wohn- und Arbeitsstandorte als auch mit Bezug auf spontane Ereignisorte und Marktbeziehungen wie Kultur- und Freizeiteinrichtungen vergrößert: „Eine Ausweitung der Reichweite bei individueller Betrachtung bedeutet gleichzeitig aber auch eine Ausweitung der Einzugsbereiche bei kollektiver Betrachtung“. Diesem Zusammenhang gilt es bei der Detailbetrachtung der einzelnen verkehrlichen Parameter Rechnung zu tragen. Holz-Rau (1997, S. 22) weist in diesem Kontext auf den Zusammenhang von kompakten Siedlungsstrukturen und einer ÖVaffinen Verkehrsmittelnutzung hin: „Dichte Bauweisen können die innerörtlichen Distanzen reduzieren. Sie schaffen gleichzeitig Nachfragekonzentrationen, die wohnungsnahe Einrichtungen tragen und ein attraktives ÖV-Angebot finanzierbar machen. Linear steigt mit der Einwohnerdichte das Nachfragepotenzial im Einzugsbereich einer Haltestelle bzw. linear sinken die Anzahl der erforderlichen Haltestellen, die zur ÖV-Bedienung zurückzulegende Strecke und damit die Fahrzeit und die Kosten“.

\section{$6 \quad$ Vorschläge für ein konkretes Erreichbarkeitsmodell}

Die Erhebung eines ÖPNV-Infrastrukturbeitrages ist - unter der Voraussetzung einer entsprechenden Rechtsgrundlage - nur dann möglich, wenn der potenzielle Nutzen für die Anlieger einer schienengebundenen ÖSPV-Infrastruktur in Städten auf kleinräumiger Ebene nachgewiesen werden kann. Zu diesem Zweck gilt es, bereits bekannte Erreichbarkeitsmodelle auf ihre Eignung zur Messung der durch eine spurgebundene ÖPNV-Infrastruktur erschlossenen Anlieger*innen auf ihre Tauglichkeit zu untersuchen und ggfs. um ein besser geeignetes Erreichbarkeitsmodell zu ergänzen.

\subsection{Erreichbarkeitsklassen der Europäischen Kommission}

Eine neue Methode zur Messbarkeit von Erreichbarkeit im ÖPNV als Voraussetzung für eine vergleichende Erreichbarkeitsanalyse europäischer Großstädte 
hat die Europäische Kommission in Rahmen ihrer Kohäsionsförderung entwickelt (European Commission 2015). Anlass für die Entwicklung der neuen Methode war die Beobachtung, dass fehlende Datenverfügbarkeiten hinsichtlich der Bevölkerungsverteilung sowie der Lage der Zugangsstellen eine vergleichende Analyse im Bereich der ÖPNV-Erreichbarkeit europäischer Großstädte erschweren. Von einer vergleichenden Erreichbarkeitsanalyse verspricht sich die Europäische Kommission einen besseren Überblick über die tatsächlichen Investitionsnotwendigkeiten als Voraussetzung für einen effizienteren Fördermitteleinsatz.

Die Europäische Kommission geht in ihren Überlegungen von fünf Erreichbarkeitsklassen aus, die sich unterscheiden je nach Bus- und Straßenbahnanbindung oder Zug- und Metroanschluss. Die höchste Erreichbarkeitsklasse (Very high frequency) wird für Haltestellen mit mehr als 10 Abfahrten/h jeweils für Busse und Straßenbahnen sowie Zug- und Metroanschluss angenommen. Alle anderen Erreichbarkeitsklassen gelten entweder für Bus und Straßenbahn oder Zug und U-Bahn. Eine hohe Erreichbarkeit (High frequency) findet sich demnach im Bereich von mehr als 10 Abfahrten, eine mittlere Erreichbarkeit (Medium frequency) gilt im Bereich von vier bis $10 \mathrm{Abfahrten} / \mathrm{h}$ und eine geringe Erreichbarkeit (Low frequency) für bis zu 4 Abfahrten/h. Keine Erreichbarkeit (No services) ist dort gegeben, wo weder eine Bus- oder Straßenbahnhaltestelle noch ein Bahnhof bzw. eine Metrostation in fußläufiger Entfernung (für Bus- bzw. Straßenbahnhaltestellen werden hier $5 \mathrm{~min}$ bzw. $417 \mathrm{~m}$ und bei Metro- und Zugstationen $10 \mathrm{~min}$ bzw. $833 \mathrm{~m}$ unterstellt). vorhanden sind. Unter Zugrundelegung dieser Erreichbarkeitsklassen hat die Europäische Kommission die ÖPNV-Erreichbarkeitsklassen von jeweils 14 europäischen Groß- sowie Mittelstädten miteinander verglichen. ${ }^{9}$

\footnotetext{
${ }^{9}$ Die Klassifizierung der Städte beruht auf der Grundlage einer neuen gemeinsamen Definition von OECD und EU. Diese identifiziert 828 (größere) urbane Zentren mit mindestens 50.000 Einwohnern in der EU (incl. Kroatien), der Schweiz, Island und Norwegen. Grundlage der Identifikation als urbanes Zentrum ist eine Bevölkerungsdichte von mehr als 1500 Einwohner*innen/qm in Siedlungen mit mindestens 50.000 Einwohner*innen, welche diese Voraussetzung erfüllen. Auf dieser Grundlage werden diejenigen urbanen Zentren als Städte klassifiziert, in denen neben einer entsprechenden politischen Zuschreibung mindestens die Hälfte der Bevölkerung innerhalb des so definierten urbanen Zentrums und wenigstens $75 \%$ der Bevölkerung des urbanen Zentrums in der Stadt lebt. Vgl. European Commission Hrsg. Cities in Europe. The new OECDEC Definition. RF 01/2012, S. 1, https://ec.europa.eu/regional_policy/en/information/ publications/regional-focus/2012/cities-in-europe-the-new-oecd-ec-definition. Zugegriffen 22.11.2017.
} 
Die höchsten ÖPNV-Erreichbarkeitsanteile (Very high und high frequency) finden sich demnach in Brüssel, Marseille und Turin mit jeweils einem Anteil von mindestens $80 \%$ der Bevölkerung, die innerhalb des urbanen Zentrums lebt. Als einzige deutsche Großstadt findet sich Berlin innerhalb einer Bandbreite von über $60 \%$ und unter $80 \%$ Bevölkerungsanteil im urbanen Zentrum. In mittelgroßen Städten finden sich die höchsten Erreichbarkeitsanteile (Very high and high frequency) erst oberhalb eines Bevölkerungsanteils von $60 \%$, der innerhalb des urbanen Zentrums lebt. Dies sind Malmö, Utrecht und Tallinn. Deutsche Städte finden sich in letzterer Kategorie überhaupt nicht (European Commission 2015, S. 4). Dieser Umstand spricht jedoch nicht gegen die beschriebene Klassifizierung von Erreichbarkeiten bzw. Erschließungsqualitäten im bzw. mit spurgebundener ÖPNV-Infrastruktur in Städten als Voraussetzung für die Einführung einer Beitragspflicht. Vielmehr erlaubt eine solche Vorgehensweise gerade die für die Einführung einer Beitragspflicht erforderliche Maßstabsbildung.

\section{2 ÖV-Güteklassen in der Schweiz}

Ein konkretes Anwendungsbeispiel für die Ermittlung und Bewertung von Erreichbarkeiten im ÖPNV als Grundlage letztlich auch für Finanzierungsentscheidungen findet sich in den vom Bundesamt für Raumentwicklung (ARE) des Eidgenossenschaftlichen Departements für Umwelt, Verkehr, Energie und Kommunikation (UVEK) veröffentlichten ÖV-Güteklassen (Bundesamt für Raumentwicklung 2011).

Seit einigen Jahren erfolgt die Ermittlung der ÖV-Güteklassen in einem automatisierten Prozess aus den Daten des elektronischen Fahrplans der Schweizerischen Transportunternehmungen (HAFAS). Dabei wird zunächst aus der Art des Verkehrsmittels - hierbei wird unterschieden in die drei Verkehrsmittelgruppen A (Bahnknoten), B (Straßenbahnen, Busse, Postautos, Rufbusse und Schiffe) sowie C (Seilbahnen) - und der Taktfolge die Haltestellenkategorie bestimmt. Anschließend wird diese mit der Entfernung zur Haltestelle zu ÖVGüteklassen kombiniert. Auf dieser Grundlage wird jede im HAFAS-Fahrplan integrierte Haltestelle einer ÖV-Güteklasse zugeordnet. Die Bestimmung der Taktfolgen erfolgt stichtagsbezogen (dabei handelt es sich um einen Werktag außerhalb der Ferienzeit und der touristischen Hochsaison) zwischen $6.00 \mathrm{Uhr}$ und 20.00 Uhr; zwecks Ermittlung der durchschnittlichen Abfahrten pro Haltestelle und Richtung wird die Anzahl der Abfahrten halbiert - unter Korrektur von Endhaltestellen sowie nur in einer Richtung angefahrenen Haltestellen. Im Anschluss daran werden für die Verkehrsmittelgruppen A und B jeweils getrennt 
die Taktfolgen berechnet und diese danach zu fünf Haltestellenkategorien verknüpft, ,wobei die Qualität der Erschließung mit steigender Kategoriennummer abnimmt“ (Bundesamt für Raumentwicklung 2013, S. 19). Als zusätzlicher Erreichbarkeitsparameter wird dann die Luftliniendistanz zur nächstgelegenen Haltestelle gemessen und auf dieser Grundlage die ÖV-Güteklasse bestimmt. Die Entfernungsradien betragen dabei 300 m, 500 m, 750 m und $1000 \mathrm{~m}$. Insgesamt entstehen so fünf ÖV-Güteklassen, die sich aus der Haltestellenkategorie und der Haltestellendistanz ergeben: Die Güteklasse A steht für eine sehr gute Erschließung, die Güteklasse B für eine gute, die Güteklasse C für eine mittelmäßige und die Güteklasse D für eine geringe Erschließung. Keine Güteklasse bedeutet eine marginale oder keinerlei ÖV-Erschließung (Bundesamt für Raumentwicklung 2013, S. 20).

\subsection{Mainzer Stellplatzsatzung mit ÖPNV-Bonus}

Ein weiteres Anwendungsbeispiel zur Bewertung von ÖPNV-Erreichbarkeiten mit finanzwirksamen Auswirkungen findet sich in der Stellplatzsatzung der rheinland-pfälzischen Landeshauptstadt Mainz (2015). Ausgehend von der Feststellung eines sowohl insgesamt rückläufigen Kfz-Stellplatzbedarfs für Wohnnutzungen als auch im Hinblick auf einzelne Stadtgebiete hat der Stadtrat beschlossen, bei der Ermittlung des notwendigen Stellplatzbedarfs künftig auch für Wohnnutzungen einen ÖPNV-Bonus zu gewähren. ${ }^{10}$ Nachdem die Landesbauordnung Rheinland-Pfalz seit 2015 in $\S 88$ den Gemeinden die Möglichkeit eröffnet, durch Satzung Vorschriften über die Zahl der notwendigen Stellplätze zu treffen, hat die Stadt Mainz mit dem Erlass der Stellplatzsatzung 2015 und insbesondere für den Regelungsbereich der Fahrradstellplätze davon in einer Weise Gebrauch gemacht, die den Anspruch auf Förderung der Verkehrsträger des Umweltverbundes mit der Vermeidung von unnötigen Baukosten sowie von Flächenverbrauch verbindet. Demnach wird ein nach Gebietszonen gestaffelter ÖPNV-Bonus gewährt, um den die Anzahl der notwendigen Stellplätze zu verringern ist. Dieser Bonus beträgt in der Gebietszone I $30 \%$, in der Gebietszone II $20 \%$ und in der Gebietszone III $10 \%$. Die parzellenscharfen Gebietszonen unterteilen sich je nach der Erschließungsqualität mit ÖPNV und hier insbesondere mit Straßenbahnen (Tab. 1).

\footnotetext{
${ }^{10}$ In der Satzungsdrucksache wird zur Begründung für die beabsichtigte Reduzierung der Kfz-Stellplatzverpflichtung auf die Erkenntnisse aus der Erhebung der SrV verwiesen.
} 
Tab. 1 Mainzer Stellplatzsatzung mit ÖPNV-Bonus

\begin{tabular}{|c|c|c|c|c|}
\hline ÖPNV-Bonus & \multicolumn{2}{|l|}{ Nur Bus } & \multicolumn{2}{|c|}{ Nur Straßenbahn (Tram) } \\
\hline \multirow[t]{3}{*}{$30 \%$} & $\begin{array}{l}\geq 12 \text { Abfahrten } \\
\text { HVZ oder }\end{array}$ & $\begin{array}{l}\text { und } \leq 10 \mathrm{~min} \\
\text { Entfernung } \mathrm{HBF}\end{array}$ & $\begin{array}{l}\geq 12 \text { Abfahrten } \\
\text { HVZ oder }\end{array}$ & $\begin{array}{l}\text { und } \leq 5 \mathrm{~min} \\
\text { Entfernung } \mathrm{HBF} \\
\text { oder }\end{array}$ \\
\hline & $\begin{array}{l}\leq 6-\geq 11 \\
\text { Abfahrten } \\
\text { HVZ oder }\end{array}$ & $\begin{array}{l}\text { und } \leq 5 \text { min Ent- } \\
\text { fernung HBF } \\
\text { oder }\end{array}$ & $\begin{array}{l}\leq 6-\geq 11 \\
\text { Abfahrten } \\
\text { HVZ oder }\end{array}$ & $\begin{array}{l}\text { und } \leq 10 \mathrm{~min} \\
\text { Entfernung } \mathrm{HBF} \\
\text { oder }\end{array}$ \\
\hline & $\begin{array}{l}\text { Fußläufige } \\
\text { Erreichbar- } \\
\text { keit Römisches } \\
\text { Theater }(600 \mathrm{~m})\end{array}$ & $\begin{array}{l}\text { und } \leq 10 \mathrm{~min} \\
\text { Entfernung } \mathrm{HBF}\end{array}$ & $\begin{array}{l}\geq 6 \text { Abfahrten } \\
\text { HVZ }\end{array}$ & $\begin{array}{l}\text { und } \leq 5 \mathrm{~min} \\
\text { Entfernung } \mathrm{HBF}\end{array}$ \\
\hline \multirow[t]{3}{*}{$20 \%$} & $\begin{array}{l}\geq 12 \text { Abfahrten } \\
\text { HVZ oder }\end{array}$ & $\begin{array}{l}\text { und } \\
\geq 11-\leq 15 \mathrm{~min} \\
\text { Entfernung } \mathrm{HBF}\end{array}$ & $\begin{array}{l}\geq 12 \text { Abfahrten } \\
\text { HVZ oder }\end{array}$ & $\begin{array}{l}\text { und } \\
\geq 6-\leq 20 \mathrm{~min} \\
\text { Entfernung } \mathrm{HBF} \\
\text { oder }\end{array}$ \\
\hline & $\begin{array}{l}\leq 6-\geq 11 \\
\text { Abfahrten } \\
\text { HVZ oder }\end{array}$ & $\begin{array}{l}\text { und } \\
\geq 6-\leq 10 \mathrm{~min} \\
\text { Entfernung } \mathrm{HBF} \\
\text { oder }\end{array}$ & $\begin{array}{l}\leq 6-\geq 11 \\
\text { Abfahrten } \\
\text { HVZ oder }\end{array}$ & $\begin{array}{l}\text { und } \\
\geq 11-\leq 15 \mathrm{~min} \\
\text { Entfernung } \mathrm{HBF} \\
\text { oder }\end{array}$ \\
\hline & $\begin{array}{l}\geq 6 \text { Abfahrten } \\
\text { HVZ }\end{array}$ & $\begin{array}{l}\text { und } \leq 5 \text { min Ent- } \\
\text { fernung } \mathrm{HBF}\end{array}$ & $\begin{array}{l}\geq 6 \text { Abfahrten } \\
\text { HVZ }\end{array}$ & $\begin{array}{l}\text { und } \leq 10 \mathrm{~min} \\
\text { Entfernung } \mathrm{HBF}\end{array}$ \\
\hline $10 \%$ & \multicolumn{4}{|c|}{$\begin{array}{l}\text { Alle übrigen ÖPNV-Angebote (Bus/Straßenbahn (Tram)) liegen in einem } \\
\text { Einzugsradius von } 300 \mathrm{~m} \text { um die ÖPNV-Haltestellen }\end{array}$} \\
\hline
\end{tabular}

Quelle: Eigene Darstellung, abgedruckt in: Mietzsch 2019

In analoger Anwendung dieses Modells auf das Anliegen der vorliegenden Arbeit könnten somit alle drei mit einem ÖPNV-Bonus in der Stadt Mainz versehenen Erreichbarkeits- bzw. Erschließungskategorien die Grundlage für eine entsprechende Beitragssatzung bilden. Diese könnte dann - ebenfalls in Analogie zur Vorgehensweise bei der Mainzer Stellplatzsatzung - je nach Qualitätsstufe (unterschieden nach Bedienungshäufigkeit, Reisezeit und Umsteigeerfordernisse) gestaffelt werden.

\section{4 ÖV-Erreichbarkeitsindex nach Mietzsch/Peter}

Zum Zwecke einer vergleichenden Bewertung verschiedener Erreichbarkeitsmodelle, um daraus Schlussfolgerungen für das aus Sicht des Verfassers am besten geeignete Modell zur Festsetzung eines ÖSPV-Infrastrukturbeitrages 


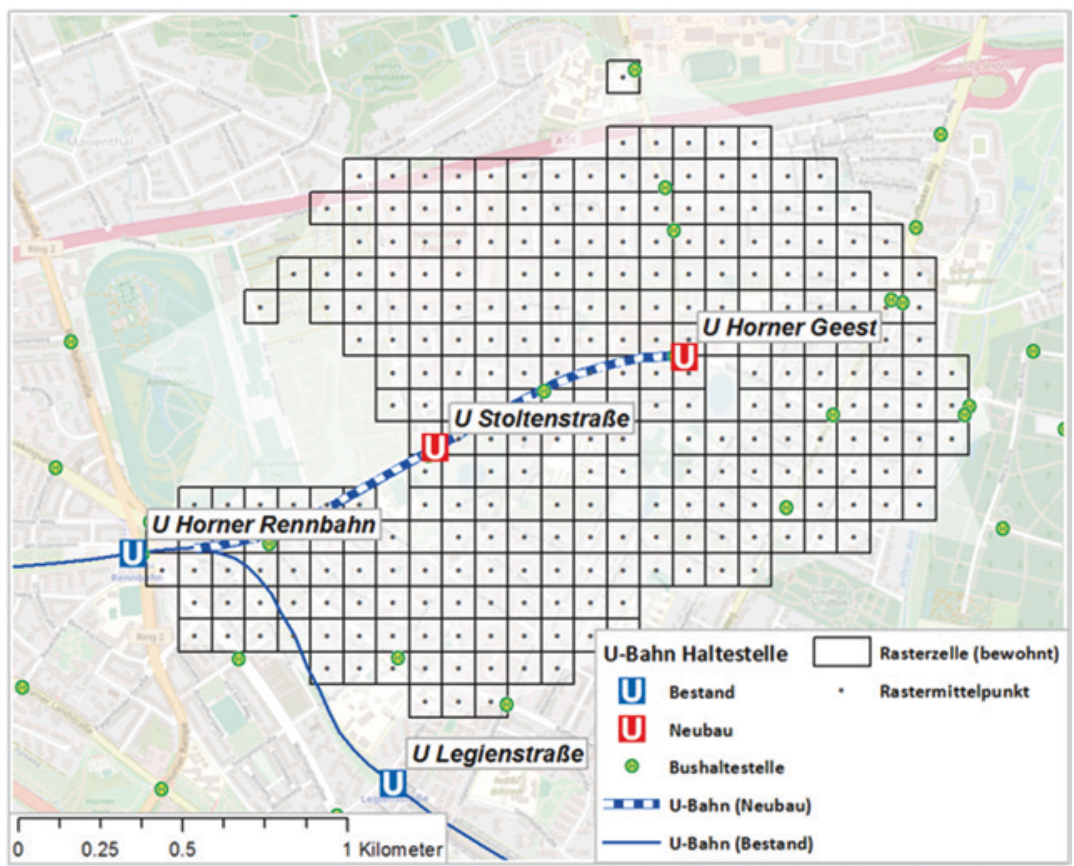

Abb. 1 Verlängerung der U4 in die Horner Geest (Planung). (Quelle: Marcus Peter, abgedruckt in: Mietzsch und Peter 2019)

ziehen zu können, erfolgt im Folgenden eine Adaption der verschiedenen Erreichbarkeitsmodelle auf die Bedingungen der Verlängerung der U4 in Hamburg. ${ }^{11}$

Im Hamburger Osten soll die bestehende U-Bahn-Linie 4 um die Haltestellen Stoltenstraße und Dannerallee verlängert werden und etwa 15.000 Einwohner*innen erschließen $^{12}$. Begründet wird das Vorhaben insbesondere mit einer wesentlichen

\footnotetext{
${ }^{11}$ Dieses Kapitel geht auf den Beitrag von Mietzsch, Oliver und Peter, Marcus. Festsetzung von Infrastrukturbeiträgen zur Finanzierung des schienengebundenen ÖPNV in Städten auf der Basis von Erreichbarkeitsindikatoren. Verkehr und Technik (1) 2019: 3-7, (2) 2019: 59-64 und (3) 2019: 93-96 zurück.

${ }^{12}$ Fortschreibungsdaten auf Rasterebene; Primärquelle Zensus (2011): https://www.zensus2011. de/SharedDocs/Aktuelles/Ergebnisse/DemografischeGrunddaten.html;jsessionid=686F0AA66 CB3DFA747B7D9CAFB2B0578.1_cid380?nn=3065474. Zugegriffen: 21.11.2018.
} 
Verbesserung des ÖPNV-Angebotes im Planungsraum. Geplant ist eine Ausfädelung hinter der Station Horner Rennbahn und eine Gesamtlänge von 1,6 km (Abb. 1). Zwischen den Stationen Jungfernstieg und Horner Rennbahn werden die U-Bahn-Linien 2 und 4 weiterhin gemeinsam geführt. Über die neue Linie werden die Stationen Stoltenstraße und Dannerallee direkt an die zentralen Umsteigeknoten Berliner Tor, Hauptbahnhof und Jungfernstieg angebunden. Daraus ergeben sich Verbindungen mit maximal einem Umstieg in das gesamte Hamburger Schienennetz. Zum Hamburger Hauptbahnhof wird die Fahrzeit 10 bis 13 min betragen. Zumindest in der Hauptverkehrszeit ist eine Taktfolge von 5 min geplant. Für die Investition werden insgesamt 465 Mio. $€$ veranschlagt ${ }^{13}$.

Erreichbarkeit wird allgemein über die Leichtigkeit definiert, mit der unterschiedliche Personen unterschiedliche Ziele erreichen können (Dalvi und Martin 1976). In diesem Zusammenhang wird implizit auch der moderne Mobilitätsbegriff adressiert. Hier wird Mobilität als die antizipierte Möglichkeit verstanden, die individuellen Bedürfnisse außerhalb der Wohnung mithilfe von Ortsveränderungen zu befriedigen. Eine realisierte Ortsveränderung wird als Verkehr bezeichnet. Folglich kann der Umfang an Mobilität jenen des messbaren Verkehrs deutlich übersteigen. Dieses Begriffsverständnis macht es auch notwendig, die Reisezeiten zu konkreten Zielen zu berücksichtigen und nicht nur die Zugänglichkeit zum öffentlichen Verkehrssystem. Ziel von Erreichbarkeitsindikatoren ist es somit, den Umfang an Mobilitätsoptionen an einem Standort zu bewerten. Folglich sind neben den sog. einfachen Erreichbarkeitsparametern, die die Entfernung zur nächsten Haltestelle oder das Fahrtenangebot an dieser bewerten, auch sog. integrierte Erreichbarkeitsparameter erforderlich, die zur Messung zusätzlicher Mobilitätsoptionen geeignet scheinen. Denn nur diese zusätzlichen Mobilitätsoptionen eignen sich als Ausgangspunkt zur Bestimmung des ÖPNV-Infrastrukturbeitrages und ermöglichen deren Rechtfertigung unter der Voraussetzung einer entsprechenden Rechtsgrundlage.

$\mathrm{Da}$ die dargestellten Ansätze der Europäischen Kommission, des Schweizerischen Bundesamts und der Landeshauptstadt Mainz Reisezeiten zu Aktivitätsgelegenheiten nur teilweise berücksichtigen, wird ein eigenes Bewertungsmodell erstellt, welches eine genauere Quantifizierung des Zusatznutzens nach Bau der neuen U-Bahn-Linie ermöglicht. Es wird davon ausgegangen, dass die Reisezeiten und Umsteigehäufigkeiten zu konkreten Zielen entscheidend für das Mobilitätsniveau der anliegenden Wohnbevölkerung und

\footnotetext{
${ }^{13}$ Alle Angaben vgl. https://dialog.hochbahn.de/u-bahn-heute-und-morgen/das-kostet-die-u4verlaengerung-auf-die-horner-geest (Zugegriffen: 21.11.2018).
} 


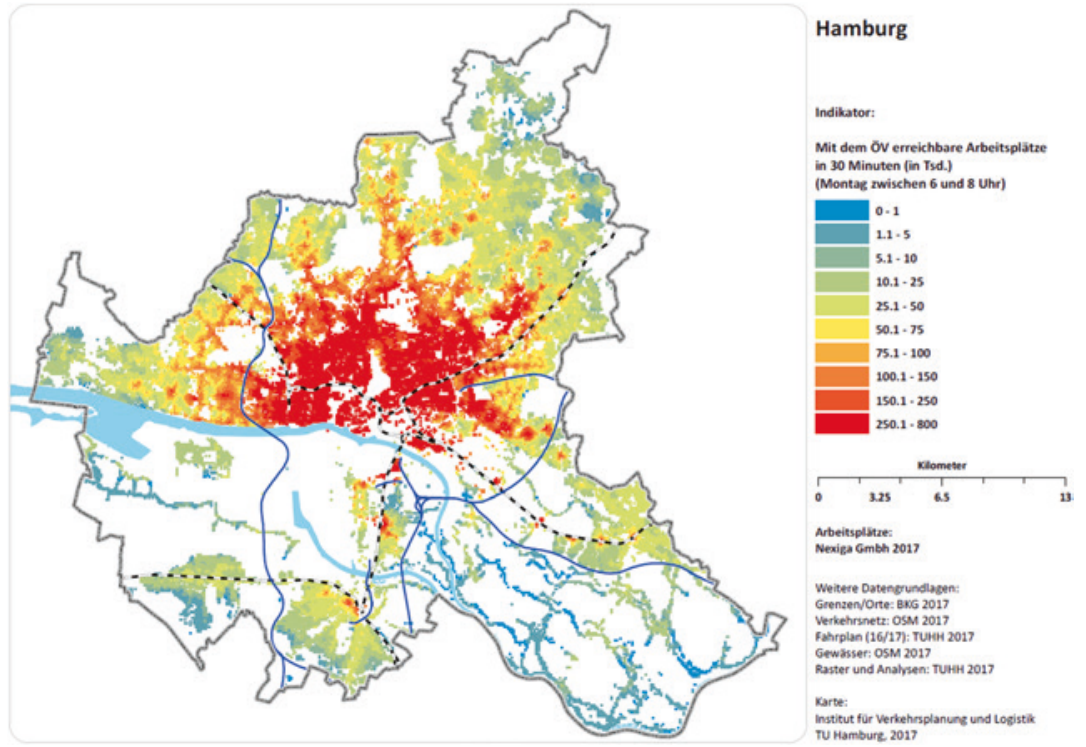

Abb. 2 Mit öffentlichen Verkehrsmitteln erreichbare Arbeitsplätze in $60 \mathrm{~min}$. (Quelle: Institut für Verkehrsplanung und Logistik TU Hamburg 2017, abgedruckt in: Mietzsch und Peter 2019)

somit als Indikatoren der Erreichbarkeit besser in der Lage sind, den Zusatznutzen abzubilden. Häufig werden Kumulationsindikatoren verwendet, um den Umfang an Mobilitätsoptionen zu bestimmen, die ein konkretes ÖPNVAngebot bietet (Boisjoly und El-Geneidy 2016). Es wird davon ausgegangen, dass ein größerer Umfang erreichbarer Ziele die Wahrscheinlichkeit erhöht, die einem Reisezweck zugrunde liegenden Bedürfnisse in einer akzeptablen Zeit zu befriedigen. Gleichwohl können auch Umstiege oder Bedienungshäufigkeiten als Raumwiderstände sowie beliebige andere Aktivitätsgelegenheiten berücksichtigt werden. Je mehr Arbeitsplätze innerhalb von 30 min erreicht werden können, als umso wahrscheinlicher wird angenommen, dass eine Person innerhalb von diesen 30 min ihren Arbeitsplatz erreichen kann (Abb. 2). Und je mehr Arbeitsplätze erreicht werden, je attraktiver ist ein Standort für Personen und Unternehmen, da von diesem Standort viele Ziele erreicht werden können. Diese verbesserte Lagegunst gegenüber Zielen drückt sich auch in steigenden Immobilienpreisen aus. Der vorgeschlagene ÖV-Erreichbarkeitsindex basiert primär auf solchen Kumulationsindikatoren. Neben Kumulationsindikatoren wird auch die 
Tab. 2 Zusammensetzung des ÖV-Erreichbarkeitsindexes. (Quelle: Marcus Peter/Oliver Mietzsch, abgedruckt in: Mietzsch und Peter 2019)

\begin{tabular}{l|l}
\hline Gewichtung (\%) & Erreichbarkeitsindikator \\
\hline 50 & Entfernung zur nächsten Bahnhaltestelle \\
\hline 7 & Erreichbare Arbeitsplätze in $30 \mathrm{~min}$ \\
8 & Erreichbare Arbeitsplätze in $60 \mathrm{~min}$ \\
8 & Erreichbare Arbeitsplätze ohne Umstieg \\
\hline 3 & Erreichbare Einwohner in $30 \mathrm{~min}$ \\
3 & Erreichbare Einwohner in $60 \mathrm{~min}$ \\
3 & Erreichbare Einwohner ohne Umstieg \\
\hline 4 & Erreichbare Freizeitgelegenheiten in $30 \mathrm{~min}$ \\
4 & Erreichbare Freizeitgelegenheiten in $60 \mathrm{~min}$ \\
4 & Erreichbare Freizeitgelegenheiten ohne \\
\hline 2 & Umstieg \\
\hline 2 & Erreichbare Einkaufsgelegenheiten in $30 \mathrm{~min}$ \\
& Erreichbare Einkaufsgelegenheiten in $60 \mathrm{~min}$ \\
\hline 100 & Erreichbare Einkaufsgelegenheiten ohne \\
\hline
\end{tabular}

Entfernung zur nächstgelegenen Bahnhaltestelle berücksichtigt, um auch die räumliche Nähe zum neuen Angebot in das Erreichbarkeitsmodell zu integrieren.

Dem vorgeschlagenen ÖV-Erreichbarkeitsindex liegen insgesamt 13 Kenngrößen bzw. Einzelindikatoren zu Grunde, diese wurden jeweils im Null- und Analysefall berechnet ${ }^{14}$. In Tab. 2 findet sich ein Überblick der je Bewertungsmethode verwendeten Einzelindikatoren. Bei der Gewichtung wird der Maximalwert jedes Einzelindikators im Untersuchungsgebiet mit $100 \%$ gewertet. Alle weiteren Indikatorwerte werden anteilig am Maximalwert berücksichtigt und anschließend anteilig in den Gesamtindikator überführt. Wenn im Untersuchungsraum maximal 500 Arbeitsplätze innerhalb einer halben Stunde erreicht werden, wird dieser Wert mit $100 \%$ gewichtet. Wenn in einer anderen Analysezelle 400 Arbeitsplätze erreicht werden, wird der Einzelindikator in dieser Zelle mit $80 \%$ gewichtet. Die hohe Gewichtung der Haltestellenentfernung reflektiert deren Bedeutung für die Attraktivität des ÖV-Angebotes.

\footnotetext{
${ }^{14}$ Nullfall $=$ Ist-Zustand; Analysefall = Nach Verlängerung der U4.
} 
Die Realdistanzen $\mathrm{zu}$ den Haltestellen wurden radiziert $^{15}$, um die relativen Abweichungen zu verringern. Anschließend wurde für jede Zelle das Vielfache vom Minimalwert berechnet und $\mathrm{zu} 100 \%$ ins Verhältnis gesetzt. Eine Realdistanz von $500 \mathrm{~m}$ bei einer minimalen Distanz von $100 \mathrm{~m}$ entspricht folglich dem fünffachen Wert bzw. 20\%. Der finale ÖV-Erreichbarkeitsindex ist eine metrische Zahl und demnach für alle Standorte unterschiedlich.

\subsection{Vergleichende Darstellung unterschiedlicher Erreichbarkeitsmodelle}

In Abb. 3 wird die Verteilung relativer Infrastrukturbeiträge räumlich dargestellt. Eine konkrete Beitragsschätzung wurde nicht vorgenommen. Gleichwohl zeigen die Ergebnisse deutlich, dass die zu erhebenden Beiträge ganz entscheidend vom verwendeten Messverfahren abhängen. Da die ÖPNV-Infrastrukturbeiträge von den Grundstückseigentümer*innen an der Strecke zu entrichten wären und keine Angaben über Anzahl und Ausstattung der Immobilien im Untersuchungsgebiet vorliegen, konnte eine Beitragszuordnung ebenfalls nicht erfolgen.

Der ÖV-Bonus nach der Mainzer Stellplatzsatzung würde eine Erhöhung der Infrastrukturbeiträge im Verlauf der neuen Strecke aufweisen, da mit der U-Bahn ein als höherwertig klassifiziertes Verkehrsmittel eingeführt würde und sich die Reisezeiten zum Hauptbahnhof erheblich verringern. Aufgrund der Berücksichtigung der Reisezeit zum Hamburger Hauptbahnhof nimmt der ÖV-Bonus mit zunehmender Reisezeit bzw. in Richtung der Endhaltestelle Dannerallee etwas ab. Insgesamt würde sich die Finanzierungsbasis dadurch verringern, dass eine Entfernung von $300 \mathrm{~m}$ zu Haltestellen deutlich unterhalb der etwa $1000 \mathrm{~m}$ liegen, wie sie auf Wegen zu Bahnhaltestellen üblicherweise maximal zurückgelegt wird (FGSV 2010).

Eine ähnliche Finanzierungsbasis ergäbe sich unter Berücksichtigung der Schweizer ÖV-Güteklassen. Es zeigt sich, dass auch Gebiete mit einer bereits sehr guten Busanbindung keine Erreichbarkeitsverbesserungen erzielen können, nachdem die U4 verlängert worden wäre. Dies betrifft die Gebiete südlich und

\footnotetext{
${ }^{15}$ Radizieren $=$ Wurzel ziehen. Realdistanzen von 1000 und $2000 \mathrm{~m}$ ergeben radizierte Werte von 31,6 und 14,0. Vor der Radizierung lag die Realdistanz von $1000 \mathrm{~m}$ fünffach über dem Vergleichswert, nach dem Radizieren nur noch in etwa zweifacher Höhe. Entsprechend erfolgt eine Glättung der Unterschiede zwischen den einzelnen Realdistanzen.
} 

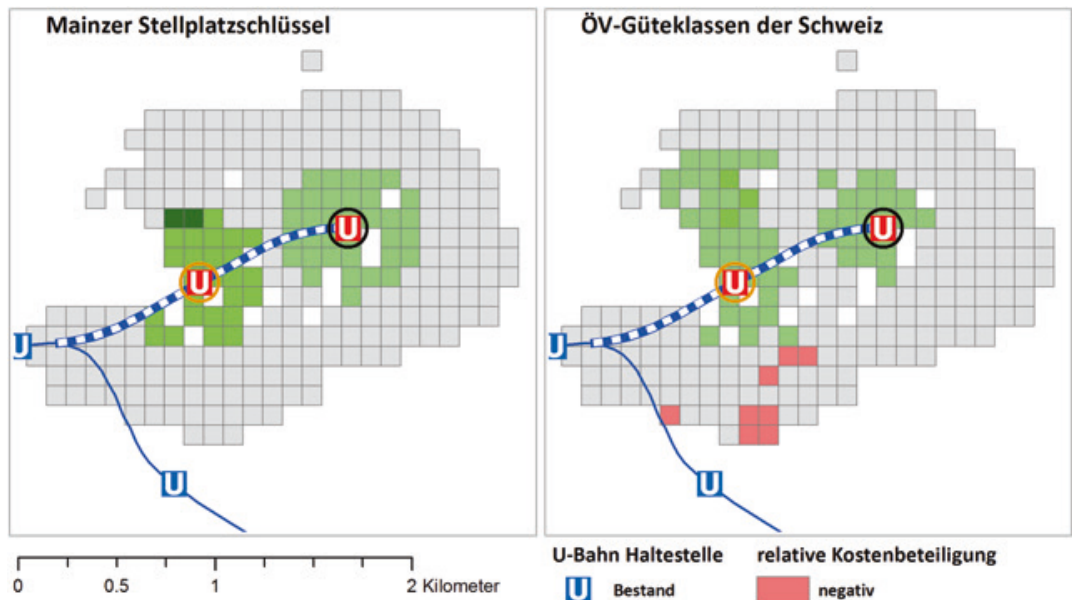

[U] Bestand negativ

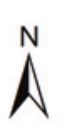

(II) Danneralle

(II) Stoltenstraße

$\square$ keine

mittel

IIII U-Bahn (Neubau) $\square$ hoch

U-Bahn (Bestand)

sehr hoch
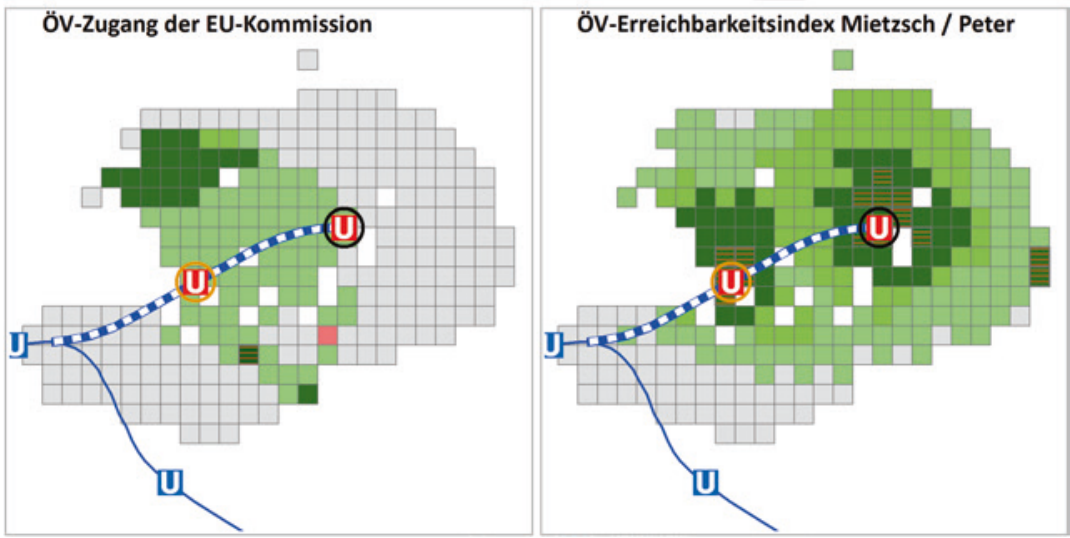

Abb. 3 Relative Finanzierungsbeteiligungen auf Basis unterschiedlicher Berechnungsgrundlagen. (Quelle: Marcus Peter, abgedruckt in: Mietzsch und Peter 2019)

östlich der Station Dannerallee. Diese werden durch die Metrobuslinien 23 und 27 bereits gut erschlossen. Dies würde $\mathrm{zu}$ der Situation führen, dass Grundstückseigentümer im direkten Stationsumfeld nicht an der Finanzierung beteiligt 
würden. Dem steht jedoch eine Beitragserhebung bei Besitzern in deutlich entfernteren Lagen im Umkreis der Station Stoltenstraße gegenüber. Nördlich der Station Stoltenstraße existieren außerdem drei Bereiche mit einem größeren Zuwachs als im direkten Haltestellenumfeld. Dies liegt daran, dass bei einer U-Bahn-Haltestelle längere Gehstrecken als akzeptabel angesehen werden als bei Bus-Haltestellen. Im südlichen Einzugsgebiet befinden sich zusätzlich einzelne Standorte, die aufgrund der Angebotsverschlechterung an der Legienstraße eine negative Entwicklung der Erreichbarkeitskennwerte aufweisen.

Die geplante U-Bahn-Verlängerung in Hamburg wird nach den Vorstellungen der EU-Kommission als Eisenbahn verstanden, die Zugänge entsprechend als Bahnhöfe deklariert. Auf Bemessungsgrundlage des ÖV-Zugangs der EU würde sich insbesondere für das Umfeld der Stoltenstraße eine Angebotsverbesserung ergeben. Die größten Zuwächse sind in relativ großer Distanz zur Haltestelle Stoltenstraße vorhanden. Im Busverkehr werden Maximaldistanzen von $417 \mathrm{~m}$ berücksichtigt, im Schienenverkehr von $833 \mathrm{~m}$. Dies bedeutet, dass Gebiete, die vor Einführung der U4 außerhalb des Einzugsgebietes einer Bushaltestelle lagen, nach Einführung der U-Bahn-Linie im Einzugsbereich einer Bahnhaltestelle liegen. Entsprechend deutlich erhöht sich das Erreichbarkeitsniveau. Gleichwohl wäre es vermutlich nicht möglich, auf Basis solcher Annahmen Finanzierungsbeiträge zu erheben. Zumal jene Eigentümer relativ am höchsten belastet wären, deren Immobilien vergleichsweise entfernt zur neuen Bahnstrecke liegen. Erschwerend kommt hinzu, dass die scharfe Begrenzung auf $833 \mathrm{~m}$ zur Schwellenbildung führt. So liegen Immobilien, deren Eigentümer einen hohen Beitrag zu entrichten hätten, direkt neben jenen, deren Besitzer keine Beiträge entrichten müssten.

Der ÖV-Erreichbarkeitsindex nach Mietzsch/Peter weist demgegenüber für die Gebiete im Umkreis der Stationen Dannerallee und Stoltenstraße umfangreiche Erreichbarkeitsgewinne aus. Alle Gebiete im Umkreis der neuen Haltestellen profitieren von der Verlängerung der U 4. Der Einfluss der Entfernung zur Haltestelle ist jedoch deutlich erkennbar. Nicht erkennbar sind die etwas geringere Reisezeit zum Hamburger Hauptbahnhof und die Innenstadt ausgehend von der Stoltenstraße. Da die Reisezeitersparnisse jedoch bei lediglich zwei Minuten liegen, muss diese Einsparung ohnehin als vernachlässigbar angesehen werden. Wesentlich bedeutsamer ist hier der deutliche Einfluss der Haltestellenentfernung sowie der größere räumliche Umfang der zu beteiligenden Nutznießer. Ein entsprechend ausgestalteter ÖSPV-Infrastrukturbeitrag wäre somit sowohl in der Lage, für viele Anrainer*innen von spurgebundener ÖPNV-Infrastruktur in Städten eine Bemessungsgrundlage für deren Finanzierungsbeteiligung zu schaffen und diese darüber hinaus in ein realistisches Verhältnis zum 
tatsächlichen Nutzen zu setzen. Mithilfe dieses ÖV-Erreichbarkeitsindex ist es möglich, differenzierter darzustellen, welchen Mehrwert das zusätzliche Angebot bietet. Dies wiederum dürfte eine nicht nur hinreichende, sondern vielmehr notwendige Voraussetzung für die gerichtsfeste Anwendung eines entsprechend ausgestalteten Finanzierungsinstrumentes sein unter der Voraussetzung einer diesbezüglichen Rechtsgrundlage.

\section{$7 \quad$ Fazit}

Ob die vorgestellten Erreichbarkeitsmodelle ausreichen, um die hohen rechtlichen Anforderungen an ein adäquates Verhältnis von Kosten und Nutzen als Voraussetzung für die Einführung einer Nutznießerbeteiligung bei der spurgebundenen ÖPNV-Infrastruktur zu erfüllen oder ob es anderer Erreichbarkeitsmodelle bedarf, bleibt so lange ungeklärt, wie es keine praktische Anwendung des vorgeschlagenen neuen Finanzierungsinstrumentes gibt. Voraussetzung hierfür ist eine gesicherte Rechtsgrundlage für die Kommunen z. B. in Form der Erweiterung des Anlagenbegriffs um den ÖPNV im BauGB, um den Neu- und Ausbau von spurgebundener ÖSPV-Infrastruktur anteilig über Beiträge der Nutznießer*innen mitfinanzieren zu können. Angesichts historisch überholter, verkehrspolitisch unausgewogener und finanztechnisch einengender Rahmenbedingungen für die Verkehrsfinanzierung ist es höchste Zeit, neue Wege zu gehen. Die Verkehrswende bedarf einer Entsprechung nicht zuletzt in rechtlicher und administrativer Hinsicht, die Mobilität und Verkehr nicht länger als Gegensätze begreift, sondern mithilfe eines integrierten Finanzierungs- und Planungsansatzes erst die Voraussetzungen dafür schafft, dass mehr Mobilität mit weniger Verkehr möglich wird (vgl. den Beitrag von Schwedes in diesem Band).

\section{Literatur}

Apel, Dieter. et. al. Flächen sparen, Verkehr reduzieren. Difu-Beiträge zur Stadtentwicklung. 1995 Berlin.

Bednarz, Liane ,Wie die Moralkritik zur Pose wird‘. Der Tagesspiegel.https://causa. tagesspiegel.de/kolumnen/liane-bednarz/wie-die-moralkritik-zur-pose-wird.html. Zugegriffen: 01.08.2020.

Boisjoly, G. und El-Geneidy, A. M. Daily fluctuations in transit and job availability: A comparative assessment of time-sensitive accessibility measures. In Journal of Transport Geography, Bd. 52, 2016: 73-81. 
Bundesamt für Raumentwicklung (Hrsg.)ÖV-Güteklassen. Berechnungsmethodik ARE (Grundlagenbericht für die Beurteilung der Agglomerationsprogramme Verkehr und Siedlung, 11.2011 Aktualisierung 02.2017, https://www.are.admin.ch/dam/are/de/ dokumente/verkehr/oev-gueteklassen-berechnungsmethodikare.pdf.download.pdf/ oev-gueteklassen-berechnungsmethodikare.pdf, https://www.are.admin.ch/dam/are/de/ dokumente/verkehr/oev-gueteklassen-berechnungsmethodikare.pdf.download.pdf/oevgueteklassen-berechnungsmethodikare.pdf. Zugegriffen: 22.11.2017.

Bundesamt für Raumentwicklung (Hrsg.)Reisezeiten und ÖV-Güteklassen. Aktualisierung der Geodaten und Karten, 08.2013, https:/www.are.admin.ch/dam/are/de/dokumente/ reisezeiten_und_oev-gueteklassen.pdf.download.pdf/reisezeiten_und_oev-gueteklassen. pdf. Zugegriffen: 22.11.2017.

Dalvi, M. Q. und Martin, K M.: The measurement of accessibility: Some preliminary results. In Transportation. Planning - Policy - Research - Practice, Bd. 5, (1) 1976, S. $17-42$.

Deutsch, Volker et. al. 2016. Integration von Stadtplanung und ÖPNV für lebenswerte Städte. Difu-Papers, Juni 2016.

European Commission Hrsg. Cities in Europe. The new OECD-EC Definition. RF 01/2012, S. 1, https://ec.europa.eu/regional_policy/en/information/publications/regional-focus/2012/ cities-in-europe-the-new-oecd-ec-definition. Zugegriffen 22.11.2017.

European Commission Hrsg. Study on urban mobility - Assessing and improving the accessibility of urban areas, https://ec.europa.eu/transport/themes/urban/news/2017-0407-study-urban-mobility-\%E2\%80\%93-assessing-and-improving-accessibility-urban_ en. Zugegriffen: 22.11.2017.

European Commission Hrsg. Measuring access to public transport in European cities. Regional Working Paper 2015 WP, https://ec.europa.eu/regional_policy/sources/ docgener/work/2015_01_publ_transp.pdfZugegriffen 22.11.2017.

Forschungsgesellschaft für Straßen- und Verkehrswesen (FGSV), Arbeitsgruppe Verkehrsmanagement (2010)(Hrsg.): Richtlinien für Lichtsignalanlagen (RiLSA); aktualisiert 2015.

Grziwotz, Herbert. In Ernst, Werner et.al. BauGB 103. EL. 2012.

Grossekettler, Heinz. Die Versorgung mit Kollektivgütern als ordnungspolitisches Problem. Volkswirtschaftliche Diskussionsbeiträge Universität Münster. Bd. 135. Manuskript eines Beitrages zum Eucken-Gedenkband des Jahrbuchs ORDO (vorläufige Fassung). Münster 1991.

Heckmann, Dirk. Gestaltungskraft und Geltungsverlust von Rechtsnormen. Tübingen 1997. Hegelau, Hans Joachim. Die rechtliche Zulässigkeit einer Nahverkehrsabgabe. Institut Finanzen und Steuern e. V. Nr. 39. Bonn 1994.

Hilgers, Hans Anton (Bearbeiter). Erschließungsbeiträge zur Finanzierung des ÖPNV. Deutscher Bundestag, Wissenschaftliche Dienste (2012) Hrsg. WD 7-3000-217/12.

Holz-Rau, Christian. Siedlungsstruktur und öffentlicher Personennahverkehr. Zur Integration des Nahverkehrsplans. Vereinigung für Stadt-, Regional und Landesplanung (SRL) Hrgs. Der Nahverkehrsplan - Neue Chance für Stadtentwicklung und Verkehrsplanung? Berlin 1997, S. 16-25.

https://www.d-copernicus.de/landueberwachung. Zugegriffen: 22.11.2017.

https://www.wirtschaftslexikon24.com/d/sprungfixe-kosten/sprungfixe-kosten.htm. Zugegriffen: 07.08.2020. 
https://www.agora-verkehrswende.de/fileadmin/Projekte/2017/Parkraummanagement/ Parkraummanagemet-lohnt-sich_Agora-Verkehrswende_web.pdf. Zugegriffen: 11.08.2020. https://www.are.admin.ch/are/de/home/verkehr-und-infrastruktur/programme-und-projekte/ agglomerationsprogramme-verkehr-und-siedlung.html. Zugegriffen: 22.11.2017.

https://dialog.hochbahn.de/u-bahn-heute-und-morgen/das-kostet-die-u4-verlaengerung-aufdie-horner-geest/ Zugegriffen: 21.11.2018.

https://wirtschaftslexikon.gabler.de/definition/positivismus-46391. Zugegriffen: 03.08.2020. https://www.zensus2011.de/SharedDocs/Aktuelles/Ergebnisse/DemografischeGrunddaten. html;jsessionid=686F0AA66CB3DFA747B7D9CAFB2B0578.1_cid380?nn=3065474. Zugegriffen: 21.11.2018.

Jarass, Hans D. und Pieroth, Bodo. Kommentar zum Grundgesetz der Bundesrepublik Deutschland. 9. Aufl., München 2007.

Klusemann, Georg-Friedrich. Kommunalabgaben - Drama ohne Ende? Hrsg. FriedrichEbert-Stiftung. Kommunalpolitische Texte Band 18. Bonn 1998.

Knieps, Günter. Knappheitsprobleme in Netzen: Was leistet die Ökonomie? Diskussionsbeiträge des Instituts für Verkehrswissenschaft und Regionalpolitik, Nr. 83. Freiburg im Breisgau 2002, https://www.econstor.eu/handle/10419/47624. Zugriff 13.10.2018.

Knoflacher, Hermann. Ingenieurwissenschaft und Verkehrstechnologie: Analyse eines Herrschaftszusammenhangs. In: Schwedes, O. et al. 2016 Hrsg. Handbuch Verkehrspolitik. 2. Aufl., Wiesbaden 2016.

Köhler, Uwe. Erreichbarkeit im Personenverkehr. In. Straßenverkehrstechnik 102011.

Landeshauptstadt Mainz. Satzung über die Herstellung und Bereitstellung von Kfz-Stellplätzen und Fahrradabstellplätzen vom 02.12.2015, Drucksache-Nr.: 1843/2015; vgl. https://Bi.mainz.de/vo0050.php?_kvonr=16807. Zugegriffen: 22.12.2017.

Lehmann, Carsten und Rodi, Hansjörg. ÖPNV in der Marktwirtschaft. Ein Ordnungsentwurf mit kritischer Würdigung der jüngsten Reformen. Diskussionspapier Nr. 5, Institut für Verkehrswissenschaft an der Universität Münster 1997.

Maak, Eckhard. Verkehrslenkende Abgabenmodelle. Gebühren und Beiträge im Dienst der Verkehrssteuerung. Dissertation. Tübingen 1998.

Mietzsch, Oliver. Nichtsteuerliche Instrumente schienengebundener ÖPNV-Infrastruktur. Dissertation, Chemnitzer Schriften für Wirtschaftsrecht Nr. 76, Lößnitz 2019.

Mietzsch, Oliver und Peter, Marcus. Festsetzung von Infrastrukturbeiträgen zur Finanzierung des schienengebundenen ÖPNV in Städten auf der Basis von Erreichbarkeitsindikatoren. Verkehr und Technik(1) 2019: 3-7, (2) 2019: 59-64 und (3) 2019: 93-96.

Sander, Ludger. Aufgaben und Einnahmen der Kommunen in der Bundesrepublik Deutschland. Münster 1987.

Schmitz, Stefan. Revolutionen der Erreichbarkeit. Gesellschaft, Raum und Verkehr im Wandel, Wiesbaden 2001.

VDV-Statistik 2018, https://www.vdv.de/statistik-jahresbericht.aspx (Zugegriffen: 11.08.2020). 
Open Access Dieses Kapitel wird unter der Creative Commons Namensnennung 4.0 International Lizenz (http://creativecommons.org/licenses/by/4.0/deed.de) veröffentlicht, welche die Nutzung, Vervielfältigung, Bearbeitung, Verbreitung und Wiedergabe in jeglichem Medium und Format erlaubt, sofern Sie den/die ursprünglichen Autor(en) und die Quelle ordnungsgemäß nennen, einen Link zur Creative Commons Lizenz beifügen und angeben, ob Änderungen vorgenommen wurden.

Die in diesem Kapitel enthaltenen Bilder und sonstiges Drittmaterial unterliegen ebenfalls der genannten Creative Commons Lizenz, sofern sich aus der Abbildungslegende nichts anderes ergibt. Sofern das betreffende Material nicht unter der genannten Creative Commons Lizenz steht und die betreffende Handlung nicht nach gesetzlichen Vorschriften erlaubt ist, ist für die oben aufgeführten Weiterverwendungen des Materials die Einwilligung des jeweiligen Rechteinhabers einzuholen.

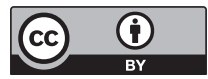

